\title{
Voltage Sensorless Control Scheme based on Extended-State Estimator for a Grid-Connected Inverter
}

\author{
Ngoc Bao Lai, Student Member, Kyeong-Hwa Kim, Member, IEEE, and Pedro Rodriguez, Fellow, IEEE
}

\begin{abstract}
This paper presents a robust voltage sensorless control scheme to eliminate the need of line-voltage sensors in an LCL-filtered grid-connected inverter system. The proposed control scheme is based on an extended-state estimator structure which consists of a state feedback regulator and an estimator. The main contribution of this study relies on the utilization of the linear matrix inequality approach and the internal model principle to synthesis a robust control scheme that equips a gridconnected inverter with the ability to withstand grid disturbances. As opposed to the existing works, the proposed control scheme can simultaneously deal with all types of grid disturbances such as grid voltage distortion, grid voltage imbalance, and grid impedance variations. The effectiveness of the proposed controller is highlighted through comparative simulation results and in-depth analyses. Experimental results are also given to validate the practicality of the proposed control scheme. ${ }^{1}$
\end{abstract}

\section{INTRODUCTION}

Recently, grid-connected inverters have been increasingly employed in distributed power generation systems (DPGS) to deliver renewable energy to the utility grid [1], [2]. Despite the fact that inverters are energy-efficient and fully controllable, many sensing devices such as the grid voltage and grid-side current sensors are required to ensure a stable inverter operation [3]. Particularly, for LCL-filtered inverters, additional sensors to measure capacitor voltage and/or inverter-side current are often employed to cope with the potentially unstable dynamics caused by LCL filter [4], [5]. This increases the cost and complexity of inverter-based DPGS [6].

Manuscript received February 27, 2019; revised June 3, 2019 and August 1, 2019; accepted November 4, 2019. This research was supported by Basic Science Research Program through the National Research Foundation of Korea (NRF) funded by the Ministry of Education (NRF2019R1A6A1A03032119), and also supported by the Spanish Ministry of Science under project ENE2017-88889-C2-1-R.

N. B. Lai is with the Research Institute on Science and Technology, LoyolaTech, Loyola University Andalusia, 41704 Seville, Spain (e-mail: nblai@uloyola.es).

K. H. Kim is with the Department of Electrical and Information Engineering, Research Center for Electrical and Information Technology, Seoul National University of Science and Technology, Seoul 01811, South Korea (e-mail:k2h1@ seoultech.ac.kr).

P. Rodriguez is with the Research Institute on Science and Technology, LoyolaTech, Loyola University Andalusia, 41704 Seville, Spain, and also with the Electrical Engineering Department, Universitat Politècnica de Catalunya, 08034 Barcelona, Spain (e-mail: prodriguez@uloyola.es, prodriguez@ee.upc.edu).

The corresponding author is Kyeong-Hwa Kim (k2h1@ seoultech.ac.kr).
Due to the better harmonic attenuation capability and smaller physical size, LCL filters are often chosen over the traditional L filters in recent years. However, the resonant characteristic of LCL filter adversely influences the performance and stability of inverter-based systems [7]. To address this problem, several damping methods have been suggested in the literature [7]-[16]. These methods fall into two main groups which are passive damping and active damping. As active damping methods are more flexible and cost-effective, they are more preferable and widely used [7]. The potential resonance caused by LCL filters can be actively damped by a full state feedback controller [8], [9]. To reduce the number of sensors, the approaches which use only the information on grid-side current or inverter-side current have been proposed in [10], [11] for the purpose of controlling the injected current stably. As another approach, the works in [12] and [13] proposed a state feedback design which guarantees the robustness of the closed-loop system by using the linear matrix inequality (LMI) approach. Other studies use the pole placement approach [14] or the concept of virtual resistor [15], [16] to deal with the inherent instability of LCL filters.

Despite the fact that these aforementioned control approaches can guarantee a stable operation of LCL-filtered inverters, they still need voltage sensors for the purpose of grid synchronization. To eliminate the need of grid voltage sensors, several line-voltage sensorless control schemes have been proposed [6], [17]-[24]. In these studies, estimators are often employed to predict the grid angular frequency and phase angle. These estimators are mainly achieved either by adaptive approaches or linear approaches. In the former case, the grid voltage is predicted by using the estimators based on the adaptive algorithms [6], [17]-[20]. In [6], a predictive controller is used to handle the injected current and an adaptive voltage estimation is used to predict the grid voltage. A control scheme which is based on the Kalman filter and sliding mode control is presented in [18]. In this work, the Kalman filter is used to observe the system states including grid voltage, and then, three decoupled sliding mode controllers (SMCs) use these estimated variables to control the inverter. As another approach, the literature [19] presents a line-voltage sensorless control scheme which is accomplished by a deadbeat control and an adaptive estimator. Because this estimator predicts not only grid voltage but also model uncertainties, a considerable performance improvement under grid voltage distortions and grid impendence variations can be observed. However, it is worth mentioning that the 
nonlinearity nature of adaptive algorithms makes it difficult to ensure and analyze the robustness of the overall system.

The latter case of line-voltage sensorless control scheme is based on linear estimators. Even though the use of a linear estimator can greatly facilitate the design and analysis of control system, only limited researches are dedicated to this area [21]-[24]. In [22], a change of variables is employed to construct an extended-state estimator. As a result of using a new set of variables, the observer dynamics and inverter parameter uncertainties can be dealt with separately. The presented experimental results prove the validity of this control scheme under the ideal voltage condition. As a different approach, virtual flux-based estimation method is proposed in [24]. Despite having considerably good performance, these approaches only address partially the issues related to voltage sensorless control of LCL-filtered inverter.

Considering the limitations of the aforementioned approaches, extended-state estimator appears to be a promising solution. Extended-state estimator utilizes internal model principle (IMP) to achieve asymptotic reference tracking and asymptotic disturbance rejection [25]. By means of using system model combined with known characteristics of disturbance, the external disturbance can be estimated. Then, this estimated disturbance can be fedback to the system to cancel out the effect of actual disturbance. Due to the effectiveness for system with limited measurement devices, the extended-state estimator has been widely used for control design and fault diagnosis [26], [27]. Recently, extended-state estimator has been proved to be feasible for voltage sensorless control of a grid-connected inverter [23]. In [23], an extendedstate observer is used to estimate grid voltage for the current control loop. Besides good performance under unbalanced grid condition, the study in [23] also reveals that the order of the closed-loop system is directly proportional to the order of the disturbance model. Thus, incorporating the harmonic terms of grid voltage into the disturbance model increases the order of the closed-loop system significantly, which poses considerable difficulty in obtaining controller and estimator gains.

Aiming to propose a complete control design framework, this paper presents a robust control scheme for an LCL-filtered grid-connected inverter with only the grid-side current measurements. Being constructed by a state feedback regulator and an extended-state observer based on the IMP, the proposed control scheme is able to effectively suppress the grid voltage disturbance such as harmonic distortions and imbalance. Moreover, a systematic design procedure based on the LMI is proposed to obtain robust and optimal gains, which ensures a stable operation of the inverter under grid impedance variations. The novelty of this paper includes the utilization and the combination of different control design approaches to achieve a robust optimal controller for grid-connected inverter with only grid-side current feedback. In particular, the extended-state estimator-based control structure, IMP, and LMI-based design approach are combined into one complete control scheme to ensure a fast response to reference, and

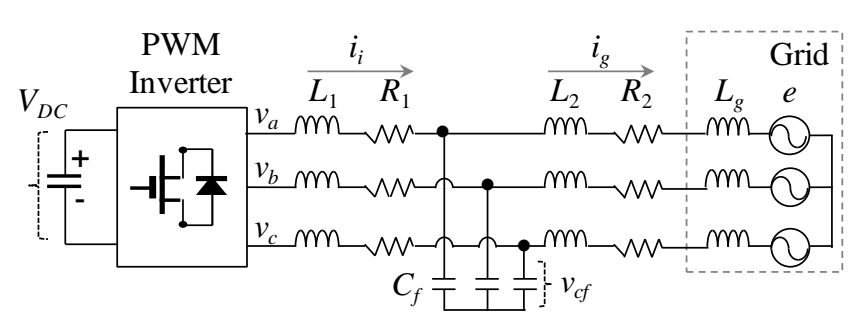

Fig. 1. Power circuit of a three-phase inverter with LCL-filters.

robustness against distorted and unbalanced grid voltage as well as grid inductance variation. To the best of the authors' knowledge, such a control scheme has not been reported in the literature yet. Additionally, theoretical analyses as well as the simulation and experimental results are given to prove the usefulness of the proposed control scheme.

\section{SYSTEM MODELING}

Fig. 1 shows the power circuit of a three-phase gridconnected inverter with LCL filters, where $R_{1}, R_{2}, L_{1}$, and $L_{2}$ represent the resistances and inductances of the filters, respectively, $C_{f}$ represents the filter capacitance, and $L_{g}$ represents the grid inductance. Since the voltage equations in the $\alpha$-axis and $\beta$-axis are independent of one another, it is reasonable to design a control scheme for one axis, and then, to apply it to the other.

From Fig. 1, the state-space equations of the inverter system can be given in the stationary reference frame as

$$
\begin{gathered}
\dot{\mathbf{x}}_{c}(t)=\mathbf{A}_{c} \mathbf{x}_{c}(t)+\mathbf{B}_{c} u_{c}(t)+\mathbf{B}_{d c} w_{c}(t) \\
y_{c}(t)=\mathbf{C}_{c} \mathbf{x}_{c}(t)
\end{gathered}
$$

where $\mathbf{x}_{c}=\left[\begin{array}{lll}i_{i} & v_{c f} & i_{g}\end{array}\right]^{T}$ with $i_{i}, v_{c f}$, and $i_{g}$ being the inverterside current, the capacitor voltage, and the grid-side current, respectively, $u_{c}=v$ is the inverter output voltage, $w_{c}=e$ is the grid voltage, and the parametric matrices are given by

$$
\begin{gathered}
\mathbf{A}_{c}=\left[\begin{array}{ccc}
-R_{1} / L_{1} & -1 / L_{1} & 0 \\
1 / C_{f} & 0 & -1 / C_{f} \\
0 & 1 /\left(L_{2}+L_{g}\right) & -R_{2} /\left(L_{2}+L_{g}\right)
\end{array}\right], \mathbf{B}_{c}=\left[\begin{array}{c}
1 / L_{1} \\
0 \\
0
\end{array}\right] \\
\mathbf{B}_{d c}=\left[\begin{array}{c}
0 \\
0 \\
-1 /\left(L_{2}+L_{g}\right)
\end{array}\right], \mathbf{C}_{c}=\left[\begin{array}{lll}
0 & 0 & 1
\end{array}\right] .
\end{gathered}
$$

For the purpose of designing a discrete controller, it is necessary to discretize the inverter system in (1) and (2). Generally, the input of the inverter system has the form of a staircase signal with sampling period $T_{s}$. Therefore, zeroorder-hold technique can be used to obtain exact discretization of (1) and (2) in the time domain as [28]

$$
\left[\begin{array}{c}
\mathbf{x}_{d i s}(k+1) \\
x_{\varphi}(k+1)
\end{array}\right]=\left[\begin{array}{cc}
\mathbf{A}_{d i s} & \mathbf{B}_{d i s} \\
\mathbf{0} & 0
\end{array}\right]\left[\begin{array}{c}
\mathbf{x}_{d i s}(k) \\
x_{\varphi}(k)
\end{array}\right]+\left[\begin{array}{l}
\mathbf{0} \\
1
\end{array}\right] u(k)+\left[\begin{array}{c}
\mathbf{B}_{d d i s} \\
0
\end{array}\right] w(k)
$$




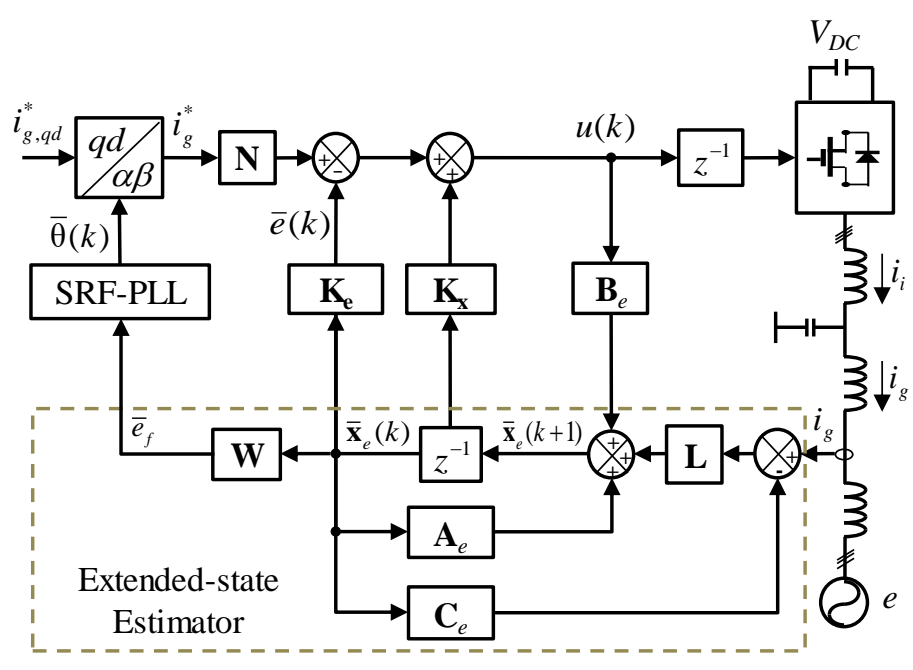

Fig. 2. Block diagram of the proposed control scheme.

$$
y(k)=\left[\begin{array}{ll}
\mathbf{C}_{c} & 0
\end{array}\right]\left[\begin{array}{c}
\mathbf{x}_{d i s}(k) \\
x_{\varphi}(k)
\end{array}\right]
$$

where $\mathbf{A}_{d i s}=e^{\mathbf{A}_{c} T_{s}}, \mathbf{B}_{d i s}=\int_{0}^{T_{s}} e^{\mathbf{A}_{c} \eta} d \eta \cdot \mathbf{B}_{c}, \mathbf{B}_{d d i s}=\int_{0}^{T_{s}} e^{\mathbf{A}_{c} \eta} d \eta \cdot \mathbf{B}_{d c}, \mathbf{0}$ is the zero matrix with appropriate dimension, the variables $\mathbf{x}_{d i s}(k), u(k), w(k), y(k)$ are the discrete-time equivalence of $\mathbf{x}_{c}(t), u_{c}(t), w_{c}(t), y_{c}(t)$, respectively, and the additional state $x_{\varphi}$ is introduced to model one sample period delay in the control input signal $u$ caused by the digital implementation. The system in (4) and (5) can be expressed in a compact form as

$$
\begin{gathered}
\mathbf{x}(k+1)=\mathbf{A x}(k)+\mathbf{B} u(k)+\mathbf{B}_{d} w(k) \\
y(k)=\mathbf{C x}(k) .
\end{gathered}
$$

\section{Proposed Voltage Sensorless Control Scheme}

This section presents the proposed control scheme. Fig. 2 shows the block diagram of the proposed control scheme which mainly consists of a state feedback regulator and an extended-state estimator. First, the design procedure for the state feedback regulator based on the LMI approach is given. Then, the extended-state estimator as well as grid synchronization is explained.

\section{A. State Feedback Regulator}

As the grid impedance varies, the parametric matrices in (3) vary accordingly. As a result, the system in (6) and (7) includes uncertain parameters. According to [29], Equation (6) can be expressed in a polytopic form as

$$
\mathbf{x}(k+1)=\mathbf{A}(\alpha) \mathbf{x}(k)+\mathbf{B} u(k)+\mathbf{B}_{d}(\alpha) w(k)
$$

where

$$
\left(\mathbf{A}, \mathbf{B}_{d}\right)(\alpha)=\sum_{j=1}^{2} \alpha_{j}\left(\mathbf{A}, \mathbf{B}_{d}\right)_{j}, \sum_{j=1}^{2} \alpha_{j}=1, \alpha_{j} \geq 0, j=1,2 .
$$

The vertex matrices in (9) are obtained by evaluating the matrices $\left(\mathbf{A}, \mathbf{B}_{d}\right)(\alpha)$ for the maximum and minimum values of $L_{g}$.

A state feedback regulator is introduced to stabilize the inverter system considering significant variations of grid impedance during operation. For the given system in (7) and (8), a state feedback regulator can be given as

$$
u(k)=\mathbf{K} \mathbf{x}(k) .
$$

The closed-loop system using the state feedback regulator can be obtained by substituting (10) into (8) as

$$
\begin{gathered}
\mathbf{x}(k+1)=(\mathbf{A}(\alpha)+\mathbf{B K}) \mathbf{x}(k)+\mathbf{B}_{d}(\alpha) w(k) \\
y(k)=\mathbf{C x}(k) .
\end{gathered}
$$

The system in (11) is quadratically stable if, and only if, the following inequalities hold as [30]

$$
\left[\begin{array}{cc}
\varepsilon^{2} \mathbf{Q} & \left(\mathbf{A}_{j} \mathbf{Q}+\mathbf{B Y}\right)^{T} \\
\mathbf{A}_{j} \mathbf{Q}+\mathbf{B Y} & \mathbf{Q}
\end{array}\right]>0 \text { and } \mathbf{Q}>0 .
$$

where $\mathbf{Q} \in \mathfrak{R}^{4 x 4}$ is a positive-definite matrix, $\varepsilon \in(0,1)$ is the decay rate which determines the convergent speed of the system states and $\mathbf{Y}=\mathbf{K Q}$.

Robust and optimal feedback gain $\mathrm{K}$ for a chosen decay rate can be obtained by solving the following LMI optimization problem:

$$
\underset{\varepsilon>0}{\operatorname{Minimize}} \mathbf{Q} \quad \text { subject to (13) . }
$$

In (14), $\mathbf{A}_{j}$ is obtained by using (11) and (12), and $\varepsilon$ is chosen decay rate. The variables $\mathbf{Y}$ and $\mathbf{Q}$ can be calculated by a LMI solver such as LMI control Toolbox in MATLAB [31]. Then, the feedback gain vector can be evaluated as $\mathbf{K}=\mathbf{Y Q}^{-1}$.

\section{B. Extended-state Estimator}

The main objective of constructing an extended-state estimator is to achieve the tracking performance as well as disturbance rejection capability without using any voltage sensors. For this purpose, the disturbances, which are the grid voltage and parameter variation, are estimated and fed back to the inverter system as a compensating control input to cancel out their effect. While these disturbances can be only compensated by applying an appropriate control input $u(k)$, both $\mathbf{B}_{d}(\alpha)$ and $w(k)$ in (6) have uncertainties. Therefore, it is necessary to introduce an equivalent component which can be applied at the control input to remove the effect on injected current by the actual disturbances [28]. As a result, applying the negative of the equivalent disturbance at control input will cancel out the effect of grid voltage and parameter variation on the system output, which is the injected current. Considering the equivalent disturbance, (8) can be rewritten as

$$
\mathbf{x}(k+1)=\mathbf{A}(\alpha) \mathbf{x}(k)+\mathbf{B}\left(u(k)+w_{e}(k)\right)
$$


where $w_{e}$ is the equivalent disturbance.

At this point, it is worth noting that although the magnitude and phase angle of $w_{e}$ are generally unknown, its frequency is identical with grid voltage frequency, as a property of a linear time-invariant system. For a three-phase system, the dominant harmonics are in frequencies of $(6 k \pm 1)^{\text {th }}$ with $\mathrm{k}$ being a non-negative integer. Since both the fundamental and harmonic voltages of the grid are sinusoidal functions of time, they can be modeled as

$$
\begin{aligned}
\dot{\mathbf{x}}_{w e c i}(t) & =\mathbf{F}_{c i} \mathbf{x}_{\text {weci }}(t) \\
w_{e c i}(t) & =\mathbf{H}_{i} \mathbf{x}_{\text {weci }}(t)
\end{aligned}
$$

where $\mathbf{x}_{\text {weci }} \in \mathfrak{R}^{2 \times 1}$ being the state vector of disturbance model

$$
\mathbf{F}_{c i}=\left[\begin{array}{cc}
0 & 1 \\
-\omega_{\mathrm{i}}^{2} & 0
\end{array}\right], \quad \mathbf{H}_{i}=\left[\begin{array}{l}
1 \\
0
\end{array}\right]^{T}
$$

and the subscript $i$ indicates the order of the component whose angular frequency is $\omega_{i}$. By using the state-space model in (16) and (17), arbitrary harmonically distorted grid voltage can be represented as

$$
\begin{aligned}
\dot{\mathbf{x}}_{w e c}(t) & =\mathbf{F}_{c} \mathbf{x}_{w e c}(t) \\
w_{e c}(t) & =\mathbf{H} \mathbf{x}_{w e c}(t)
\end{aligned}
$$

where

$$
\mathbf{x}_{\text {wec }}=\left[\begin{array}{c}
\mathbf{x}_{\text {wec } 1} \\
\vdots \\
\mathbf{x}_{\text {wec }} \\
\vdots
\end{array}\right], \mathbf{F}_{c}=\left[\begin{array}{cccc}
\mathbf{F}_{c 1} & & & \\
& \ddots & & \\
& & \mathbf{F}_{c i} & \\
& & & \ddots
\end{array}\right], \mathbf{H}=\left[\begin{array}{c}
\mathbf{H}_{1}^{T} \\
\vdots \\
\mathbf{H}_{i}^{T} \\
\vdots
\end{array}\right]^{T} .
$$

The discrete-time representation of (19) and (20) can be given as

$$
\begin{gathered}
\mathbf{x}_{w e}(k+1)=\mathbf{F}_{d} \mathbf{x}_{w e}(k) \\
w_{e}(k)=\mathbf{H} \mathbf{x}_{w e}(k)
\end{gathered}
$$

where $\mathbf{F}_{d}=e^{\mathbf{F}_{c} T_{s}}$. To construct the extended-state estimator, the grid voltage model in (22) and (23) can be augmented with the inverter model in (7) and (15) as

$$
\begin{gathered}
{\left[\begin{array}{c}
\mathbf{x}(k+1) \\
\mathbf{x}_{w e}(k+1)
\end{array}\right]=\left[\begin{array}{cc}
\mathbf{A}(\alpha) & \mathbf{B H} \\
\mathbf{0} & \mathbf{F}_{d}
\end{array}\right]\left[\begin{array}{c}
\mathbf{x}(k) \\
\mathbf{x}_{w e}(k)
\end{array}\right]+\left[\begin{array}{c}
\mathbf{B} \\
\mathbf{0}
\end{array}\right] u(k)} \\
y(k)=\left[\begin{array}{ll}
\mathbf{C} & \mathbf{0}
\end{array}\right]\left[\begin{array}{c}
\mathbf{x}(k) \\
\mathbf{x}_{w e}(k)
\end{array}\right] .
\end{gathered}
$$

Equations (24) and (25) can be concisely expressed as

$$
\begin{gathered}
\mathbf{x}_{e}(k+1)=\mathbf{A}_{e}(\alpha) \mathbf{x}_{e}(k)+\mathbf{B}_{e} u(k) \\
y(k)=\mathbf{C}_{e} \mathbf{x}_{e}(k) .
\end{gathered}
$$

The estimator which predicts the system states and grid voltage from inverter reference voltage $u(k)$ and grid-side current measurement $y(k)$ can be given as

$$
\overline{\mathbf{x}}_{e}(k+1)=\mathbf{A}_{e}(\alpha) \overline{\mathbf{x}}_{e}(k)+\mathbf{B}_{e} u(k)+\mathbf{L}\left(\mathbf{C}_{e} \overline{\mathbf{x}}_{e}(k)-y(k)\right)
$$

where $\overline{\mathbf{x}}_{e}(k)$ denotes the estimated state and $\mathbf{L}$ is the estimator gain. The dynamics of the estimation error $\tilde{\mathbf{x}}_{e}(k)=\mathbf{x}_{e}(k)-\overline{\mathbf{x}}_{e}(k)$ can be derived by subtracting (28) from (26) as

$$
\tilde{\mathbf{x}}_{e}(k+1)=\left(\mathbf{A}_{e}(\alpha)+\mathbf{L} \mathbf{C}_{e}\right) \tilde{\mathbf{x}}_{e}(k) .
$$

For the purpose of obtaining a robust and optimal $\mathrm{L}$, the procedure used to design $\mathbf{K}$ in the previous section is applied to (29). The stability condition for the estimator in (28) can be given as

$$
\left[\begin{array}{cc}
\varepsilon_{e}^{2} \mathbf{Q}_{e} & \mathbf{Q}_{e} \mathbf{A}_{e j}+\mathbf{Y}_{e} \mathbf{C}_{e} \\
\left(\mathbf{Q}_{e} \mathbf{A}_{e j}+\mathbf{Y}_{e} \mathbf{C}_{e}\right)^{T} & \mathbf{Q}_{e}
\end{array}\right]>0 \text { and } \mathbf{Q}_{e}>0
$$

where $\mathbf{Y}_{e}=\mathbf{Q}_{e} \mathbf{L}$, and $\varepsilon_{e} \in(0,1)$ is the convergent speed of the estimator states. The matrices $\mathbf{Q}_{e}$ and $\mathbf{Y}_{e}$ can be calculated by solving the following LMI optimization:

$$
\underset{\varepsilon_{e}>0}{\operatorname{Minimize}} \mathbf{Q}_{e} \quad \text { subject to (30). }
$$

Then, the estimator gain is $\mathbf{L}=\mathbf{Q}_{e}^{-1} \mathbf{Y}_{e}$. Even though the regulator and the estimator are individually stable, the closedloop stability is not guaranteed as a result of uncertain parameters. Moreover, it is impossible to implement an estimator with varying parameters included in (28). Therefore, it is desirable to find fixed parametric matrices for the estimator, which together with $\mathbf{K}$ and $\mathbf{L}$, guarantees the stability of the closed-loop system. For this purpose, the closed-loop system which includes the regulator, the estimator, and the feedback grid voltage $\bar{e}(k)$ can be derived from (10), (15), (28), and Fig. 2 as

$$
\begin{aligned}
{\left[\begin{array}{c}
\mathbf{x}(k+1) \\
\overline{\mathbf{x}}_{e}(k+1)
\end{array}\right]=} & {\left[\begin{array}{cc}
\mathbf{A}(\boldsymbol{\alpha}) & \mathbf{B}\left(\mathbf{K}_{x}-\mathbf{K}_{e}\right) \\
-\mathbf{L C} & \mathbf{A}_{e}+\mathbf{B}_{e}\left(\mathbf{K}_{x}-\mathbf{K}_{e}\right)+\mathbf{L} \mathbf{C}_{e}
\end{array}\right]\left[\begin{array}{c}
\mathbf{x}(k) \\
\overline{\mathbf{x}}_{e}(k)
\end{array}\right] } \\
& +\left[\begin{array}{c}
\mathbf{B} \\
\mathbf{B}
\end{array}\right] \mathbf{N} r(k)+\left[\begin{array}{c}
\mathbf{B} \\
\mathbf{0}
\end{array}\right] w_{e}(k)
\end{aligned}
$$

where $\mathbf{K}_{x}=\left[\begin{array}{ll}\mathbf{K} & \mathbf{0}\end{array}\right], \mathbf{K}_{e}=\left[\begin{array}{ll}\mathbf{0} & \mathbf{H}\end{array}\right], r$ is the reference current, and $\mathbf{N}$ is a feedforward gain.

The proposed complete design procedure including the previous design process to determine the set of stable estimator parameters is shown in Fig. 3. It is worth noticing that the grid inductance $L_{g e}$ serves as a virtual value to check the stability of the system in (32) on each iteration. By using the proposed procedure, the values for $L_{g}$ which results in a stable closed-loop system can be determined. The underlying 


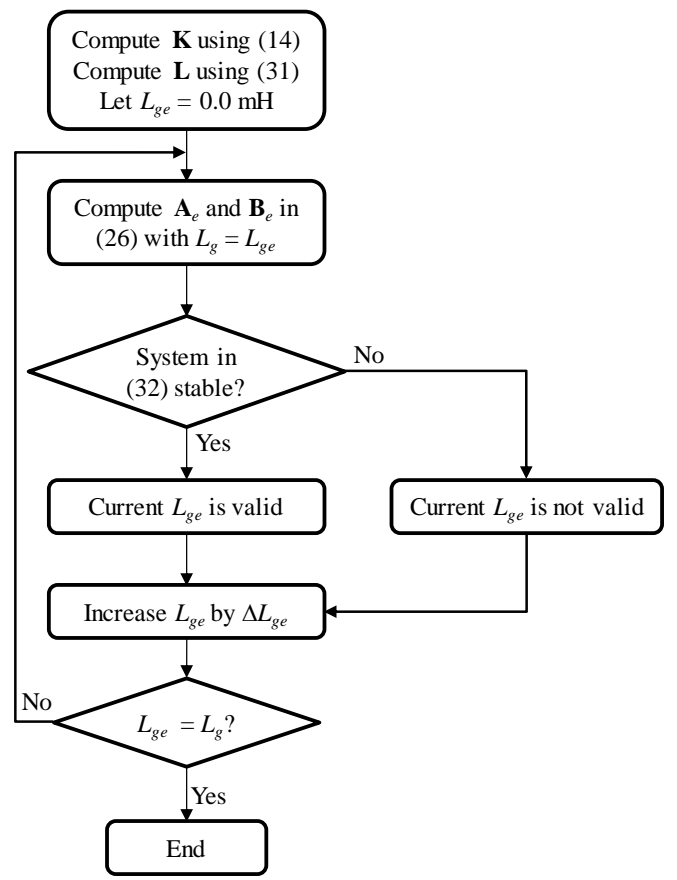

Fig. 3. Proposed design procedure of determining grid impedance values in the implementation of extended-state estimator to ensure a stable closed-loop system.

TABLE I

PARAMETERS OF THE THREE-PHASE INVERTER

\begin{tabular}{clc}
\hline \hline Symbol & \multicolumn{1}{c}{ Quantity } & Value \\
\hline$P$ & Nominal inverter power & $2.5 \mathrm{kVA}$ \\
$E$ & Nominal grid line-line voltage & $220 \mathrm{~V}$ \\
$f_{g}$ & Nominal grid frequency & $60 \mathrm{~Hz}$ \\
$V_{D C}$ & DC-link voltage & $400 \mathrm{~V}$ \\
$R_{1} / R_{2}$ & Filter resistance & $0.5 / 0.3 \Omega$ \\
$L_{1} / L_{2}$ & Filter inductance & $1.7 / 0.9 \mathrm{mH}$ \\
$C_{f}$ & Filter capacitance & $4.5 \mu \mathrm{F}$ \\
& & $0.0 \mathrm{mH}$ \\
$L_{g}$ & Grid inductance: & Min. \\
& & Max. \\
\hline
\end{tabular}

principle of the proposed procedure is based on the stability conditions of a discrete-time polytopic uncertain linear timeinvariant system [32]. These conditions state that the system in (32) is robustly stable in the uncertain domain (12) if, and only if, the stability is guaranteed at the vertexes of the uncertain domain. As a result, for each valid $L_{g e}$, an estimator which results in a stable closed-loop system can be constructed.

To demonstrate the proposed procedure, a regulator and an extended-state estimator are designed for a three-phase inverter system whose parameters are given in Table I. For the given system, the grid voltage is assumed to be polluted with $5^{\text {th }}, 7^{\text {th }}$, and $11^{\text {th }}$ harmonics. To accomplish the first two steps of the procedure, the decay rates are chosen as $\varepsilon=0.6$ and $\varepsilon_{e}=0.985$. In order to choose proper decay rates, it is worth bearing in mind that the convergent speed of system states is increased as the decay rate is decreased. On the other hand, the stability margin is decreased as the decay rate is decreased. As a result, selecting smaller decay rates yields faster transient responses and smaller stability margin. Using $\mathbf{K}$ and $\mathbf{L}$



Fig. 4. Eigenvalue map of the closed-loop system when $L_{g}$ varies from 0 to $105 \mathrm{mH}$.
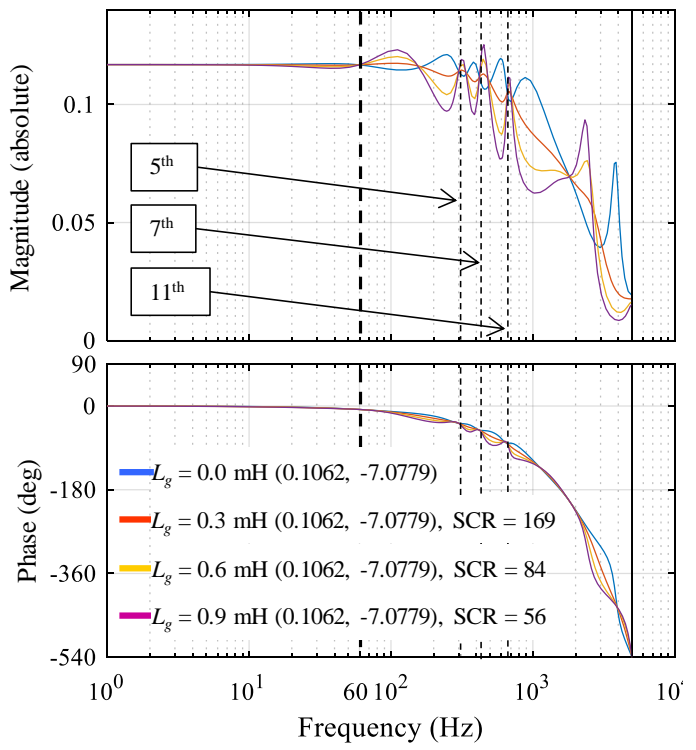

Fig. 5. Frequency responses of the closed-loop system in (32) when $N=1$, $w_{e}=0$, and $L_{g}=\{0.0,0.3,0.6,0.9\} \mathrm{mH}$. Values in parentheses indicate the magnitude gain and phase delay of corresponding response at $60 \mathrm{~Hz}$.

determined in the first two steps, the remaining steps can be proceeded with $\Delta L_{g e}=0.01 \mathrm{mH}$ to determine valid values of $L_{g e}$ which is $L_{g e} \in[0.2,0.9] \mathrm{mH}$.

Fig. 4 shows the eigenvalue map of the closed-loop system in (32) with different values of $L_{g}$. It can be observed that the eigenvalues move toward the boundary of the unit circle as the grid inductance increases. In fact, the closed-loop system reaches the stability boundary at $L_{g}=105 \mathrm{mH}$.

Nevertheless, the eigenvalues remain within the unit circle for the predefined values of $L_{g}$, which indicates the robustness of the proposed control scheme under parameter variations.

\section{Reference Tracking and Grid Synchronization}

Considering the complete control input shown in Fig. 2, the inverter state-space equation can be extracted from (32) as

$\mathbf{x}(k+1)=\mathbf{A}(\boldsymbol{\alpha}) \mathbf{x}(k)+\mathbf{B} \mathbf{K} \overline{\mathbf{x}}(k)-\mathbf{B} \bar{w}_{e}(k)+\mathbf{B N} r(k)+\mathbf{B} w_{e}(k) .(33)$

It is clearly shown in (33) that the inverter states are independent of grid voltage in the steady state when the 
estimated disturbance is equal to the real disturbance $\bar{w}_{e \infty}=w_{e \infty}$. In fact, feeding back estimated disturbance can compensate not only for the grid voltage but also for the effect of parameter variation. That is, as shown in Fig. 5, the gain and phase of the closed-loop system at the specified frequencies are fixed for different values of $L_{g}$. Oftentimes, the grid strength is conveniently indicated by short-circuit ratio (SCR) as

$$
S C R=\frac{1}{L_{g} \omega_{g}} \cdot \frac{q}{\sqrt{1+q^{2}}} \cdot \frac{E^{2}}{P}
$$

with $q$ being $X / R$ ratio of the grid. Since the gain and phase of the closed-loop system are independent of the grid voltage and inductance in the steady state, $\mathbf{N}$ can be chosen such that the closed-loop system has unity gain and zero phase delay at the fundamental frequency. For the inverter system given in Table I, the feedforward gain $\mathbf{N}$ is

$$
\mathbf{N}=\frac{1}{0.1062} \angle-\left(-7.0779^{\circ}\right)=9.4162 \angle 7.0779^{\circ} \text {. }
$$

In addition to the grid voltage, the estimated disturbance contains also the compensation component for parameter variation. However, despite this compensation part, the angle of estimated disturbance is nearly the same with that of the grid voltage as will be shown in later sections. Therefore, the angle of estimated disturbance at fundamental frequency can be used to determine the grid phase angle. Since the estimator also acts as a band-pass filter, the estimated disturbance at fundamental frequency is free of harmonics. Therefore, only the conventional phase-locked loop [33] is sufficient to identify the grid voltage angle. The fundamental component of estimated disturbance is

$$
\bar{e}_{f}=\mathbf{W} \overline{\mathbf{x}}_{e}
$$

where $\mathbf{W}=\left[\begin{array}{lll}\mathbf{0}^{1 \times 4} & -1 & \mathbf{0}^{1 \times 7}\end{array}\right]$.

In the steady state, when $\bar{w}_{e \infty}=w_{e \infty}$, Equation (33) can be written as

$$
\mathbf{x}(k+1)=\mathbf{A}(\boldsymbol{\alpha}) \mathbf{x}(k)+\mathbf{B K} \overline{\mathbf{x}}(k)+\mathbf{B N} r(k) .
$$

Equation (37) indicates that the closed-loop system is not affected by the grid voltage in the steady state. Furthermore, the influence of the variation in grid impedance is also taken into consideration as an input parameter in III.A and III.B. As a result, the proposed control scheme is capable of dealing with grid voltage disturbance as well as grid impedance variation.

\section{SIMULATION RESULTS}

To validate the proposed control scheme, the simulations based on PSIM software have been carried out. The system parameters are described in TABLE 1. Decay rates and parametric matrices of the estimator given in Section III are used to synthesize the proposed control scheme. In addition to
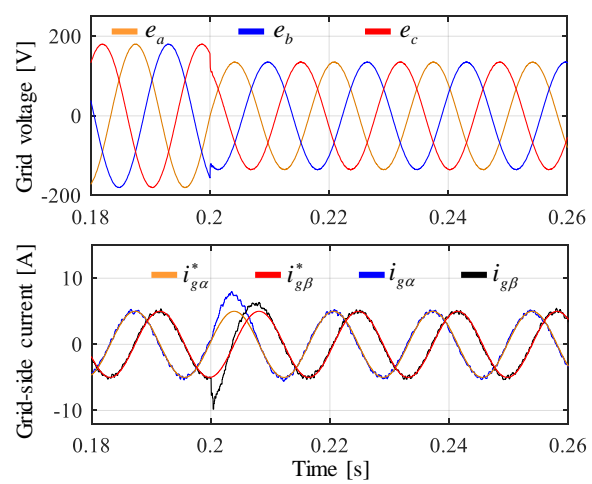

(a)


(b)

Fig. 6. Simulation results under grid voltage dip of $25 \%$ at $t=0.2 \mathrm{~s}$. (a) Control scheme presented in [23]. (b) Proposed control scheme.

the ideal grid voltage, unbalanced and distorted grid voltages are also employed to validate the performance of the proposed control scheme. This abnormal grid voltage has $20 \%$ reduction in magnitude in one phase and contains the $5^{\text {th }}$, the $7^{\text {th }}$, and the $11^{\text {th }}$ harmonics with the magnitude of $10 \%, 10 \%$, and $5 \%$ with respect to the nominal grid voltage, which yields the total harmonic distortion (THD) value of $15 \%$.

To highlight the effectiveness of the proposed control scheme, the controller presented in [23] is adopted for the comparison purpose. Fig. 6 shows the simulation results under grid voltage dip of $25 \%$ for the control scheme presented in [23] and the proposed control scheme. It can be observed that in the event of a sudden reduction in magnitude of grid voltage, both control schemes exhibit similar short transience in injected current, taking about $10 \mathrm{~ms}$ to bring the inverter system back to the steady state.

Fig. 7 shows the comparison results under unbalanced and distorted grid voltages for the control scheme presented in [23] and the proposed control scheme. As can be seen in Fig. 7a, as soon as the voltage disturbance is superimposed on the ideal voltage at $\mathrm{t}=0.2 \mathrm{~s}$, the grid-side currents become unacceptably distorted. The distorted current brings about noticeable steady-state error in the estimated voltage, which is more clearly shown in the phase error between the grid phase angle and the estimated angle. On the contrary, as shown in Fig. $7 \mathrm{~b}$, the proposed controller can effectively remove the effect of grid voltage disturbance on the injected current after a transient period of $15 \mathrm{~ms}$. Such a good disturbance rejection 

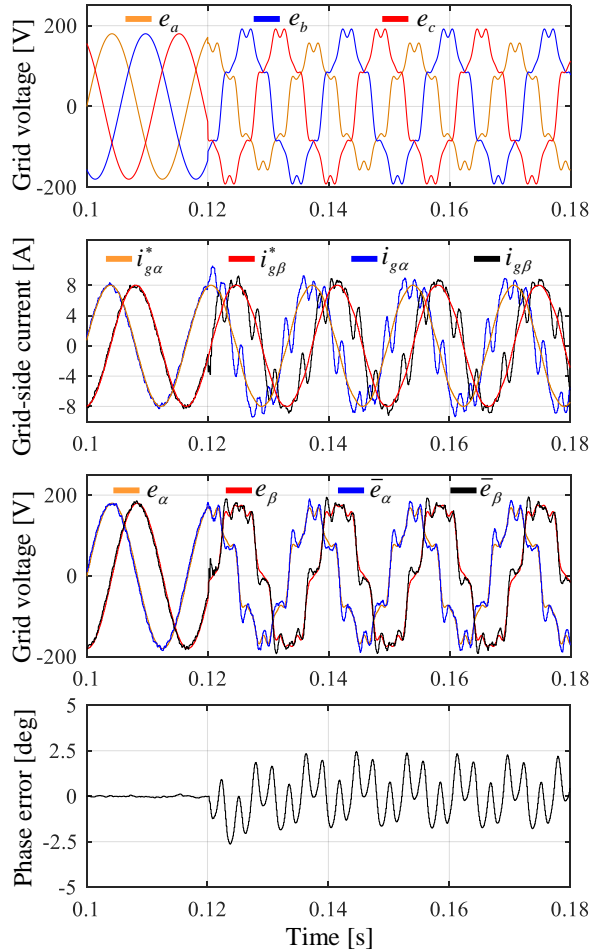

(a)
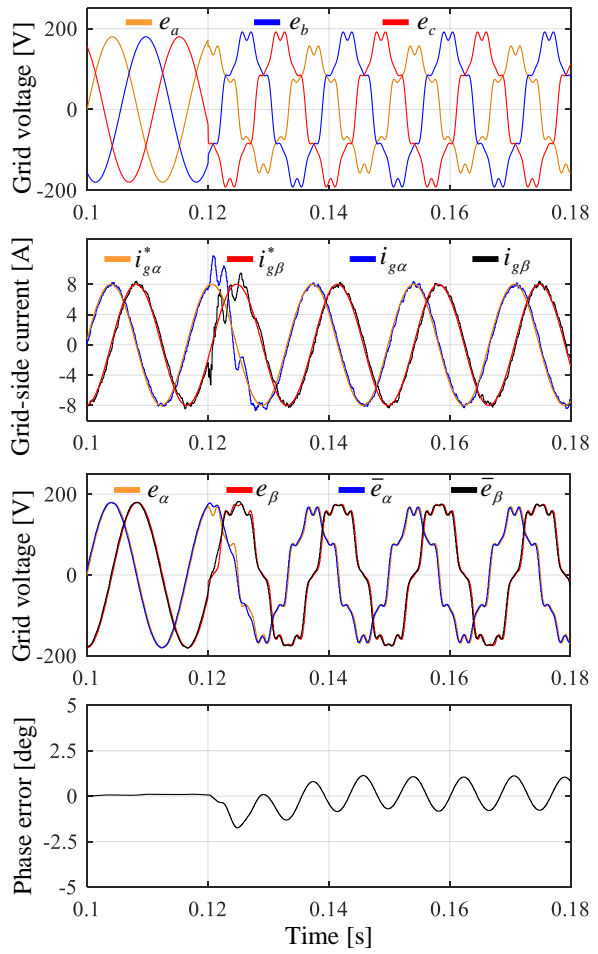

(b)

Fig. 7. Simulation results for control and estimation performance under the grid voltage change at $t=0.12 \mathrm{~s}$. (a) Control scheme presented in [23]. (b) Proposed control scheme.

capability of the proposed control scheme is achieved by the incorporation of the disturbance model in the controller.

Fig. 8 compares the performance of the two control schemes considering a sudden change in grid impedance at
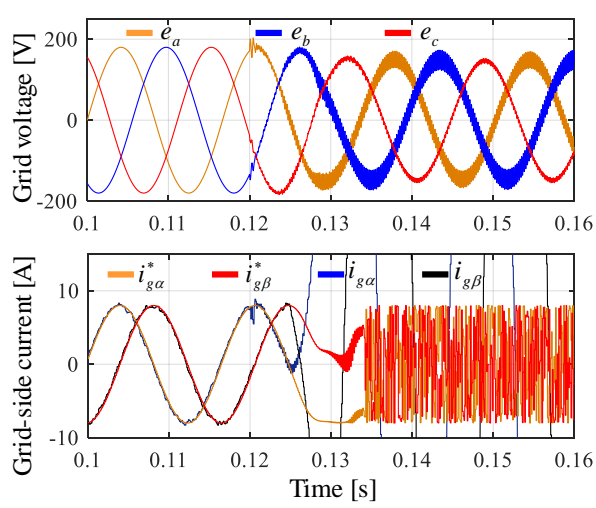

(a)

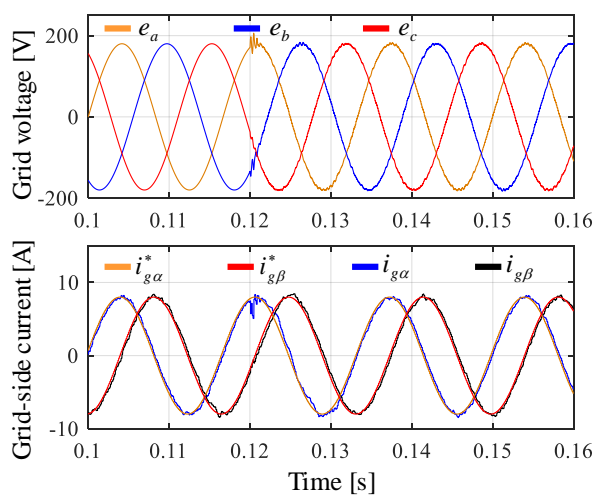

(b)

Fig. 8. Simulation results for control performance under the grid impedance change at $t=0.12$ s. (a) Control scheme presented in [23]. (b) Proposed control scheme.

$0.12 \mathrm{~s}$. As can be observed from Fig. 8a, the controller in [23] is unable to stabilize the inverter system under such a condition. However, as shown in Fig. 8b, such variation in grid impedance unnoticeably affects the inverter operation when the proposed control scheme is used. Indeed, the inverter currents only encounter a relatively short transient period. This property of the proposed controller is achieved by using LMI approach to consider the grid impedance variation in the controller design.

In comparison to the existing controller, the proposed scheme provides better control performance in case of grid harmonic voltage and parameter variation. The shortcoming in dealing with harmonic disturbance in the existing controller comes from the fact that the proportional resonant (PR) controller and the estimator contain only the fundamental frequency term [23]. Therefore, the existing approach is not capable of rejecting harmonic voltage. Moreover, the grid inductance variation was not considered in [23]. Indeed, the robustness against parameter variation in [23] is just byproduct effect rather than a predefined design criterion. On the other hand, as clearly shown in Section III, both the harmonic voltage and grid induction variation are considered as design criteria in the proposed control scheme. Particularly, the parameter variation is considered in the proposed scheme by means of polytopic modeling and LMI-based design approach. Also, the grid voltage disturbance is modeled by using the IMP as shown from (19) to (25). As a result, the 

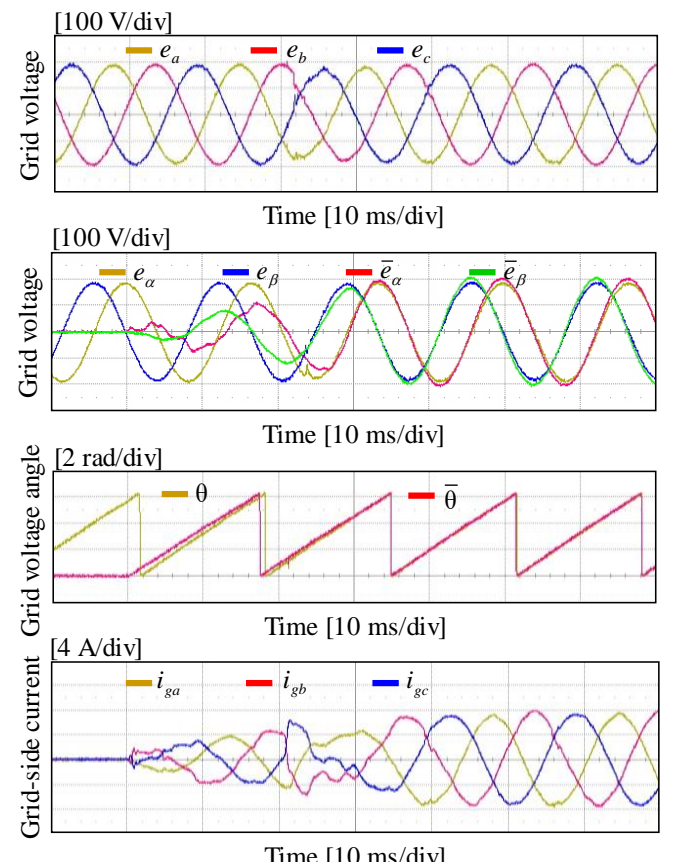

Time $[10 \mathrm{~ms} / \mathrm{div}]$

Fig. 9. Experimental results of the proposed control scheme during start-up process.
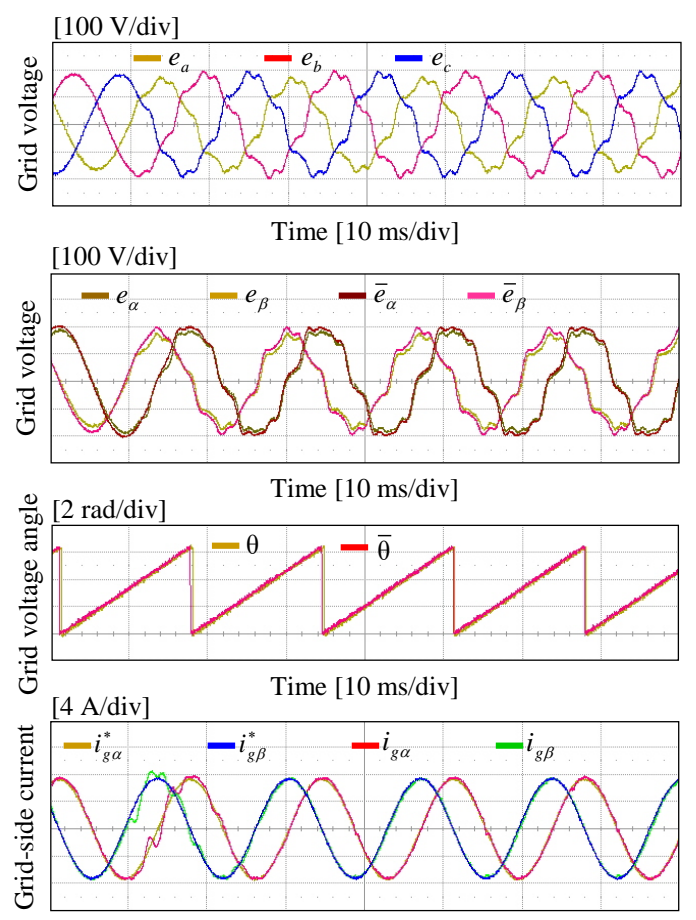

Time $[10 \mathrm{~ms} / \mathrm{div}]$

Fig. 10. Experimental results of the proposed control scheme when the grid voltage changes from the ideal condition to unbalanced and distorted one.

proposed control scheme can withstand both the grid impedance variation and grid voltage disturbance. The ability to deal simultaneously with different types of grid disturbances sets the proposed control scheme apart from the existing methods.


Fig. 11. Experimental results of the proposed control scheme when the reference current undergoes a step change.

\section{EXPERIMENTAL RESULTS}

To further validate the practicality of the proposed control scheme, the experiments using a three-phase $2.5 \mathrm{kVA}$ prototype grid-connected inverter have been performed. The prototype grid-connected inverter is implemented using an intelligent power module (IPM) PM50RL1A120 with the turnon and turn-off dead times of $1 \mu$ s within the DSP. The system parameters, the control gains, and the grid conditions are the same with those used in the simulations. The entire control scheme is implemented using 32-bit floating-point DSP TMS320F28335 with clock frequency of $150 \mathrm{MHz}$. The sampling and switching frequencies are chosen as $10 \mathrm{kHz}$. To emulate the grid, a three-phase programmable AC power source (PACIFIC Power Source 320-ASX) is connected to the filters through a transformer (PACIFIC M99211).

Fig. 9 shows experimental waveforms of the proposed control scheme during the start-up process which is one of the most critical tasks faced by voltage sensorless control schemes. As can be seen from Fig. 9, it takes about $30 \mathrm{~ms}$ for the estimated grid voltages to reach the actual values. Such transience can also be obtained from the estimated grid voltage angle. The waveforms of grid-side currents confirm a stable inverter operation during the start-up process.

Fig. 10 shows the experimental results of the proposed control scheme when the ideal grid voltages are suddenly changed into unbalanced and distorted voltages and Fig. 11 shows the experimental results when there is a step change in reference currents under unbalanced and distorted grid. All the experimental results are well matched with the simulation waveforms in Fig. 7. It is confirmed from these experimental results that the proposed voltage sensorless control scheme which uses only the grid-side current measurements operates stably as well as effectively even in such a severe grid environment.

As also seen in Fig. 9 and Fig. 10, the estimated grid voltages well assemble the actual grid voltages. However, there is small difference in magnitude between the actual and 

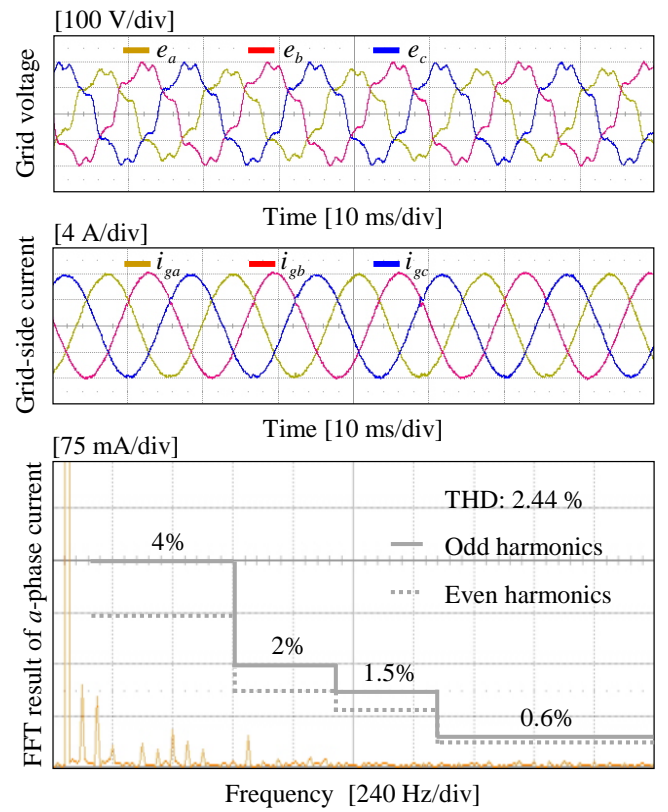

Fig. 12. Experimental results for steady-state response of the proposed control scheme under unbalanced and distorted grid voltage.

the estimated voltage because of the imperfection of the experimental system. It should be also noted that such steadystate mismatch in estimated voltage does not affect the estimated grid angle and tracking performance of the proposed control scheme.

To investigate the quality of injected currents in an experimental way, Fig. 12 shows the experimental results of the proposed control scheme under unbalanced and distorted grid voltages. In spite of the abnormal grid voltages, the gridside currents are considerably sinusoidal. In fact, according to the FFT result of $a$-phase current, the harmonic contents of injected currents well satisfy the limits specified by the grid interconnection regulation IEEE 1547 with the THD value of only $2.44 \%$.

\section{CONCLUSION}

This paper has presented a voltage sensorless control scheme for a three-phased grid-connected inverter. The proposed control scheme not only controls the inverter system stably under grid impedance variation but also ensures the high-quality injected current by using only the grid-side current sensors. Structurally, the proposed scheme mainly consists of a state feedback regulator and an extended-state estimator. Furthermore, the IMP and LMI approach have been employed to address different types of grid disturbance. Unlike the existing methods, this study proposes a systematic framework for designing a controller which is able to cope with grid voltage distortion as well as grid impedance variations at the same time. Comparative simulations under different operating conditions have shown significant improvements in control performance by using the proposed scheme. Moreover, the various experimental results based on a prototype grid-connected inverter have been presented to confirm the feasibility of the proposed control approach.

\section{REFERENCES}

[1] A. M. Bouzid, J. M. Guerrero, A. Cheriti, M. Bouhamida, P. Sicard, and M. Benghanem, "A survey on control of electric power distributed generation systems for microgrid applications," Renew. Sustain. Energy Rev., vol. 44, pp. 751-766, Apr. 2015.

[2] Y. Deng, Y. Tao, G. Chen, G. Li, and X. He, "Enhanced power flow control for grid-connected droop-controlled inverters with improved stability," IEEE Trans. Ind. Electron., vol. 64, no. 7, pp. 5919-5929, Jul. 2017.

[3] J. C. Vasquez, J. M. Guerrero, M. Savaghebi, J. Eloy-Garcia, and R. Teodorescu, "Modeling, analysis, and design of stationary-referenceframe droop-controlled parallel three-phase voltage source inverters," IEEE Trans. Ind. Electron., vol. 60, no. 4, pp. 1271-1280, Apr. 2013.

[4] J. Dannehl, F. W. Fuchs, and P. B. Thogersen, "PI state space current control of grid-connected pwm converters with LCL filters," IEEE Trans. Power Electron., vol. 25, no. 9, pp. 2320-2330, Sep. 2010.

[5] J. R. Massing, M. Stefanello, H. A. Grundling, and H. Pinheiro, "Adaptive current control for grid-connected converters with LCL filter," IEEE Trans. Ind. Electron., vol. 59, no. 12, pp. 4681-4693, Dec. 2012.

[6] K. H. Ahmed, A. M. Massoud, S. J. Finney, and B. W. Williams, "A modified stationary reference frame-based predictive current control with zero steady-state error for LCL coupled inverter-based distributed generation systems," IEEE Trans. Ind. Electron., vol. 58, no. 4, pp. 1359-1370, Apr. 2011.

[7] M. Liserre, F. Blaabjerg, and S. Hansen, "Design and control of an LCLfilter-based three-phase active rectifier," IEEE Trans. Ind. Appl., vol. 41, no. 5, pp. 1281-1291, Sep./Oct. 2005.

[8] L. A. Maccari, J. R. Massing, L. Schuch, C. Rech, H. Pinheiro, R. C. L. F. Oliveira, and V. F. Montagner, "LMI-based control for gridconnected converters with LCL filters under uncertain parameters," IEEE Trans. Power Electron., vol. 29, no. 7, pp. 3776-3785, Jul. 2014.

[9] N. Panten, N. Hoffmann, and F. W. Fuchs, "Finite control set model predictive current control for grid-connected voltage-source converters with LCL filters: A study based on different state feedbacks," IEEE Trans. Power Electron., vol. 31, no. 7, pp. 5189-5200, Jul. 2016.

[10] J. Dannehl, M. Liserre, and F. W. Fuchs, "Filter-based active damping of voltage source converters with LCL filter," IEEE Trans. Ind. Electron., vol. 58, no. 8, pp. 3623-3633, Aug. 2011.

[11] W. Yao, Y. Yang, X. Zhang, F. Blaabjerg, and P. C. Loh, "Design and analysis of robust active damping for LCL filters using digital notch filters," IEEE Trans. Power Electron., vol. 32, no. 3, pp. 2360-2375, Mar. 2017.

[12] I. J. Gabe, V. F. Montagner, and H. Pinheiro, "Design and implementation of a robust current controller for VSI connected to the grid through an LCL filter," IEEE Trans. Power Electron., vol. 24, no. 6, pp. 1444-1452, Jun. 2009

[13] M. S. Sadabadi, A. Haddadi, H. Karimi, and A. Karimi, "A robust active damping control strategy for an LCL-based grid-connected DG unit," IEEE Trans. Ind. Electron., vol. 64, no. 10, pp. 8055-8065, Oct. 2017.

[14] D. Perez-Estevez, J. Doval-Gandoy, A. G. Yepes, and O. Lopez, "Positive- and negative-sequence current controller with direct discretetime pole placement for grid-tied converters with LCL filter," IEEE Trans. Power Electron., vol. 32, no. 9, pp. 7207-7221, Sep. 2017.

[15] J. Scoltock, T. Geyer, and U. K. Madawala, "A model predictive direct current control strategy with predictive references for MV gridconnected converters with LCL-filters," IEEE Trans. Power Electron., vol. 30, no. 10, pp. 5926-5937, Oct. 2015. 
[16] V. Miskovic, V. Blasko, T. M. Jahns, A. H. C. Smith, and C. Romenesko, "Observer-based active damping of LCL resonance in gridconnected voltage source converters," IEEE Trans. Ind. Appl., vol. 50, no. 6, pp. 3977-3985, Nov. 2014.

[17] K. H. Ahmed, A. M. Massoud, S. J. Finney, and B. W. Williams, "A modified stationary reference frame-based predictive current control with zero steady-state error for LCL coupled inverter-based distributed generation systems," IEEE Trans. Ind. Electron., vol. 58, no. 4, pp. 1359-1370, Apr. 2011.

[18] R. Guzman, L. G. de Vicuna, M. Castilla, J. tomas Miret, and J. de la Hoz, "Variable structure control for three-phase LCL-filtered inverters using a reduced converter model," IEEE Trans. Ind. Electron., 2017.

[19] Y. A-R. I. Mohamed, M. A. Rahman, and R. Seethapathy, "Robust linevoltage sensorless control and synchronization of LCL-filtered distributed generation inverters for high power quality grid connection," IEEE Trans. Power Electron., vol. 27, no. 1, pp. 87-98, Jan. 2012.

[20] J. Kukkola and M. Hinkkanen, "State observer for grid-voltage sensorless control of a converter equipped with an LCL filter: direct discrete-time design," IEEE Trans. Ind. Appl., vol. 52, no. 4, pp. 31333145, Jul./Aug. 2016.

[21] S. G. Jorge, J. A. Solsona, and C. A. Busada, "Control scheme for a single-phase grid-tied voltage source converter with reduced number of sensors," IEEE Trans. Power Electron., vol. 29, no. 7, pp. 3758-3765, Jul. 2014.

[22] B. Wang, Y. Xu, Z. Shen, Z. Jibin, C. Li, and H. Liu, "Current control of grid-connected inverter with LCL filter Based on extended-state observer estimations using single sensor and achieving improved robust observation dynamics," IEEE Trans. Ind. Electron., vol. 64, no. 7, pp. 5428-5439, Jul. 2017.

[23] R. Fantino, C. A. Busada, and J. A. Solsona, "Observer-Based gridvoltage sensorless synchronization and control of a VSI-LCL tied to an unbalanced grid," IEEE Trans. Ind. Electron., in press, doi: 10.1109/TIE.2018.2868255.

[24] N. F. Roslan, J. A. Suul, J. Rocabert, and P. Rodriguez, "A comparative study of methods for estimating virtual flux at the point of common coupling in grid-connected voltage source converters with LCL filter," IEEE Trans. Ind. Appl., vol. 53, no. 6, pp. 5795-5809, Nov.-Dec. 2017.

[25] C. D. Johnson, "Accommodation of external disturbances in linear regulator and servomechanism problems," IEEE Trans. Autom. Control, vol. 16, no. 6, pp. 635-644, Dec. 1971.

[26] J. Chen, R. J. Patton, and H. Zhang, "Design of unknown input observers and robust fault detection filters," Int. J. Control, vol. 63, no. 1, pp. 85-105, 1996.

[27] W. Chen, J. Yang, L. Guo and S. Li, "Disturbance-Observer-Based Control and Related Methods-An Overview," IEEE Transactions on Industrial Electronics, vol. 63, no. 2, pp. 1083-1095, Feb. 2016.

[28] G. F. Franklin, J. D. Powell, and M. Workman, Digital Control of Dynamic Systems, 3rd ed., California, Addison Wesley Longman, 1998.

[29] S. Boyd, L. El Ghaoui, E. Feron, and V. Balakrishnan, Linear matrix inequalities in system and control theory. Philadelphia, PA, USA: SIAM Studies in Applied Mathematics, 1994.

[30] N. B. Lai and K. H. Kim, "Robust control scheme for three-phase gridconnected inverters with LCL-filter under unbalanced and distorted grid conditions," IEEE Trans. Energy Convers., vol. 33, no. 2, pp. 506-515, Jun. 2018.

[31] P. Gahinet, A. menirovskii, A. J. Laub, and M. Chilali, LMI Control Toolbox User's Guide. Natick, MA, USA: The Math Works, Inc., 1995.

[32] M. C. de Oliveira, J. Bernussou, and J. C. Geromel, "A new discretetime robust stability condition," Syst. Control Lett., vol. 37, no. 4, pp. 261-265, Jul. 1999.

[33] S. Golestan, J. M. Guerrero, and A. Abusorrah, "MAF-PLL with phaselead compensator," IEEE Trans. Ind. Electron., vol. 62, no. 6, pp. 36913695, Jun. 2014.



Ngoc Bao Lai received the B.S. degree in electrical engineering from Danang University of Science and Technology, Da Nang, Vietnam, in 2014, and the M.S. degree in electrical and information engineering at Seoul National University of Science and Technology, Seoul, Korea, in 2017. He is currently pursuing a Ph.D. degree in electrical engineering at Loyola University Andalucia.

His research interests include robust control, power electronics, and distributed generation systems.

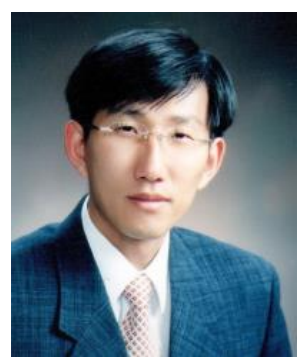

Kyeong-Hwa Kim was born in Seoul, Korea, in 1969. He received his B.S. degree from Hanyang University, Seoul, Korea, in 1991; and his M.S. and Ph.D. degrees from the Korea Advanced Institute of Science and Technology (KAIST), Daejeon, Korea, in 1993 and 1998, respectively, all in Electrical Engineering. From 1998 to 2000, he was a Research Engineer with Samsung Electronics Company, Korea, where he was engaged in the research and development of $\mathrm{AC}$ machine drive systems. From 2000 to 2002, he was a Research Professor with KAIST. From August 2010 to August 2011, he was a Visiting Scholar with the Virginia Polytechnic Institute and State University (Virginia Tech), Blacksburg, VA, USA. Since August 2002, he has been with the Seoul National University of Science and Technology, Seoul, Korea, where he is presently working as a Professor. His current research interests include AC machine drives, the control and diagnosis of power systems, power electronics, renewable energy, and DSP-based control applications. Professor Kim is a Member of the Korean Institute of Power Electronics (KIPE) and IEEE.

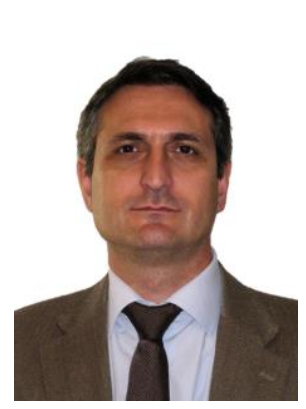

Pedro Rodriguez received his M.Sc. and Ph.D. degrees in electrical engineering from the Technical University of Catalonia (UPC), Spain. $\mathrm{He}$ was a postdoc researcher at the CPES, Virginia Tech, US, at the Department of Energy Technology, Aalborg University (AAU), Denmark and at the MIT Energy Initiative (MITie), Boston, US. He was a co-supervisor of the Vestas Power Program, Denmark (2007 2011). He was a director of technology in Modern Power Systems in Abengoa Research (2011-2017). From 2006, he is the head of the research centre Renewable Electrical Energy Systems (SEER) at the UPC. From 2017, he is a full professor at the Loyola University Andalucia (LOY), Spain, where he leads the research programme on Intelligent Energy Systems from LOYOLATECH, the research institute on science and technology at LOY.

$\mathrm{He}$ is in the list of Highly Cited Researchers in Engineering (2015-2018) published by Clarivate. He has co-authored one Wiley-IEEE book, over 100 papers in ISI technical journals, and around 300 papers in conference proceedings. He is the holder of 16 licensed patents. He has participated in more than 50 projects with industrial partners and several EU projects. Dr. Rodriguez is a IEEE Fellow for his contributions in the control of distributed generation. $\mathrm{He}$ is an Associate Editor of the IEEE Transaction on Power Electronics and the IEEE Journal on Emerging and Selected Topics on Power Electronics. He is a member of the Sustainability and Renewable Energy Committee of the IEEE Industry Application Society and the Renewable Energy Systems Technical Committee of the IEEE Industrial Electronics Society. His research interests include intelligent energy systems, distributed generation, and rural electrification. 